\section{Surfactant adsorption and aggregate structure at silica nanoparticles: Effects of particle size and surface modification $\dagger$}

\author{
Bhuvnesh Bharti, ${ }^{a}$ Jens Meissner, ${ }^{a}$ Urs Gasser ${ }^{b}$ and Gerhard H. Findenegg ${ }^{* a}$ \\ Received 20th March 2012, Accepted 13th April 2012 \\ DOI: $10.1039 / \mathrm{c} 2 \mathrm{sm} 25648 \mathrm{~g}$
}

The influence of particle size and a surface modifier on the self-assembly of the nonionic surfactant $\mathrm{C}_{12} \mathrm{E}_{5}$ at silica nanoparticles was studied by adsorption measurements and small-angle neutron scattering (SANS). Silica nanoparticles of diameter 13 to $43 \mathrm{~nm}$ were synthesized involving the basic amino acid lysine. A strong decrease of the limiting adsorption of $\mathrm{C}_{12} \mathrm{E}_{5}$ with decreasing particle diameter was found. To unveil the role of lysine as a surface modifier for the observed size dependence of surfactant adsorption, the morphology of the surfactant aggregates assembled on pure siliceous nanoparticles (Ludox-TMA, $27 \mathrm{~nm}$ ) and their evolution with increasing lysine concentration at a fixed surfactant-to-silica ratio was studied by SANS. In the absence of lysine, the surfactant forms surface micelles at silica particles. As the concentration of lysine is increased, a gradual transition from the surface micelles to detached wormlike micelles in the bulk solution is observed. The changes in surfactant aggregate morphology cause pronounced changes of the system properties, as is demonstrated by turbidity measurements as a function of temperature. These findings are discussed in terms of particle surface curvature and surfactant binding strength, which present new insight into the delicate balance between the two properties.

\section{Introduction}

Surfactant adsorption onto colloidal particles is of eminent importance to technological processes in which colloidal stability or detergency plays a role. ${ }^{1}$ Surfactant adsorption onto hydrophilic surfaces can be regarded as a surface aggregation process, reminiscent of micelle formation in solution. ${ }^{2-9}$ When the anchoring of the surfactant heads to the surface is weak, as in the case of nonionic surfactants at oxide surfaces, the morphology of surface aggregates may depend both on the anchoring strength $^{10,11}$ and on the curvature of the adsorbing surface. ${ }^{12-18}$ For instance, for the surfactant penta(ethyleneglycol) monododecylether $\left(\mathrm{C}_{12} \mathrm{E}_{5}\right)$ it was recently found that discrete surface micelles are formed on silica nanoparticles, ${ }^{14,16}$ although flat bilayer aggregates are preferred at planar silica surfaces. ${ }^{6,7}$ At even weaker anchoring energies, surface micelles may be disfavoured against micelles in solution, implying that little or no

${ }^{a}$ Technische Universität Berlin, Institut für Chemie, Stranski Laboratorium, TC 7, Strasse des 17. Juni 124, D-10623 Berlin Germany. E-mail: findenegg@chem.tu-berlin.de; Fax: +49 30314 26602, Tel: +493031424171

${ }^{b}$ Paul Scherrer Institut, 5232 Villigen, Switzerland. E-mail: urs.gasser@ psi.ch; Fax: +41 56 3102939; Tel: +41 563103229

$\dagger$ Electronic supplementary information (ESI) available: Characterization of Ludox-TMA silica nanoparticles; analysis of SANS data; SAXS for temperature variation experiments. See DOI: $10.1039 / \mathrm{c} 2 \mathrm{sm} 25648 \mathrm{~g}$ adsorption occurs, as in the case of dodecyl maltoside $\left(\beta-\mathrm{C}_{12} \mathrm{G}_{2}\right)$ at silica nanoparticles. ${ }^{\mathbf{1 4}}$

Here, we study the influence of particle size and surface modification on the adsorption of the surfactant $\mathrm{C}_{12} \mathrm{E}_{5}$ at silica nanoparticles. The particles were synthesized by a modified Stöber method ${ }^{19}$ yielding particles of narrow size distribution down to the 10 to $50 \mathrm{~nm}$ size range which was of interest in this study. In this method, the basic amino acid lysine is used instead of ammonia as the catalyst for the hydrolysis of the silica precursor. For the resulting Lys-Sil particles ${ }^{19}$ it was found that the adsorption isotherm of $\mathrm{C}_{12} \mathrm{E}_{5}$ exhibits a pronounced dependence on particle size. To assess the influence of lysine on the surface energy and the adsorption of the surfactant at the silica particles we also investigated the adsorption of lysine onto pure siliceous silica nanoparticles (Ludox-TMA) and used small angle neutron scattering (SANS) to elucidate changes in surfactant self-assembly when adding increasing amounts of lysine to the silica dispersion. Based on these results we discuss the effects of surface curvature and anchoring strength of the surfactant heads on the adsorption of $\mathrm{C}_{12} \mathrm{E}_{5}$ at silica nanoparticles.

\section{Results}

\subsection{Characterization of silica nanoparticles}

Results of the characterization of three Lys-Sil materials by small-angle X-ray scattering (SAXS), nitrogen adsorption and TEM are shown in Fig. 1. The SAXS profiles of the three samples 

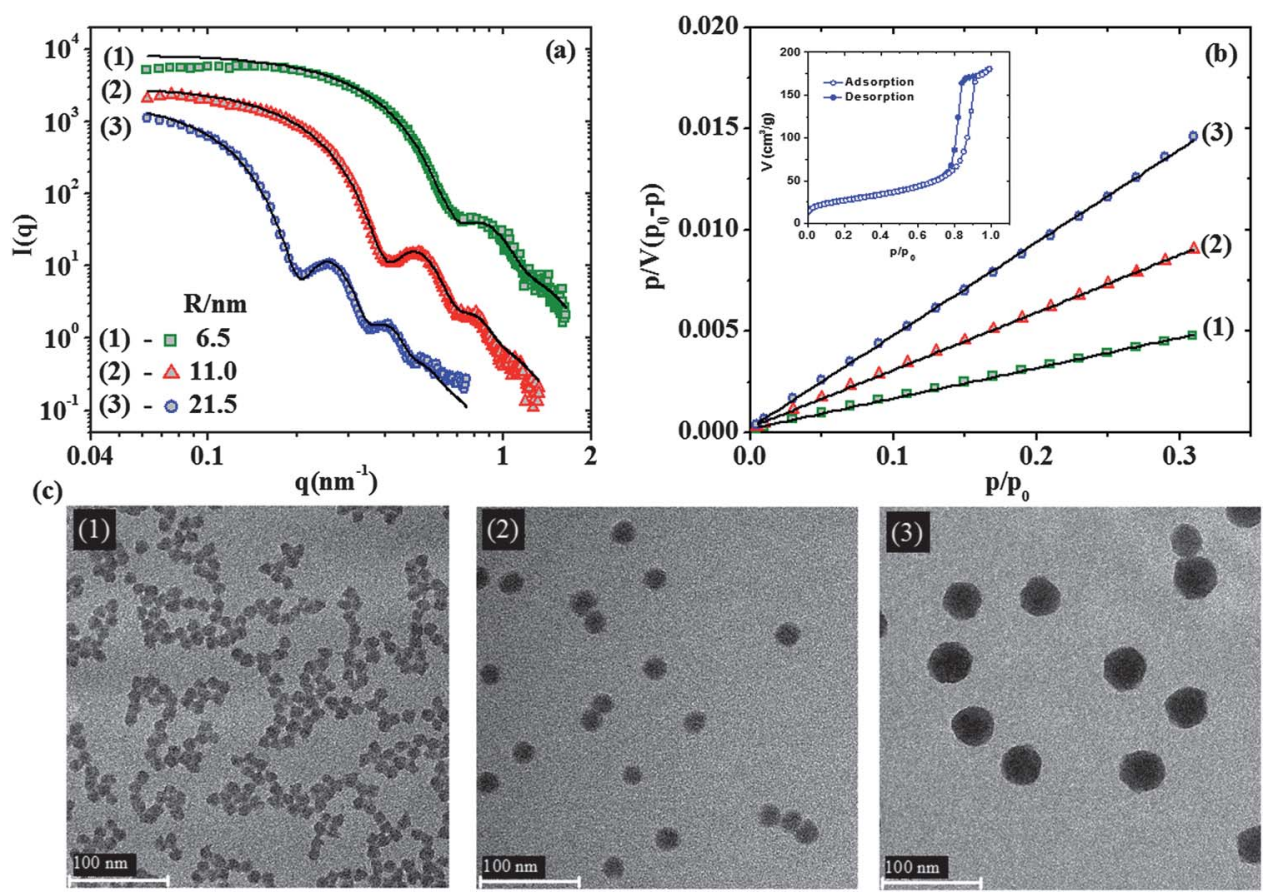

Fig. 1 Characterization of three Lys-Sil silica (1), (2) and (3): (a) experimental SAXS profiles and fits to the points according to form factor of spheres dispersed in a medium. (b) BET-plot of $\mathrm{N}_{2}$ adsorption isotherms (the inset is the measured complete isotherm for Lys-Sil-3 silica particles). (c) TEM images of the three Lys-Sil nanoparticles, shown at the same magnification.

(Fig. 1a) can be represented by the form factor model of spherical particles having a log-normal size distribution. Values of the mean particle radius $R$ and polydispersity $s$ of the three Lys-Sil materials are given in Table 1. The systematic deviations from the experimental $I(q)$ at low values of the scattering vector $q$ can be attributed to repulsive long-range interactions between the charged particles at $\mathrm{pH} 9$. These interparticle features are of no relevance for the mean particle size and size distribution.

The specific surface area of the silica sols was determined from the nitrogen adsorption isotherms by the BET method in a range of relative pressures $p / p_{0}$ from 0.05 to 0.3 (Fig. 1b). The resulting values $a_{\mathrm{BET}}$ are given in Table 1 and compared with the geometric surface area per unit mass, $a_{\mathrm{geo}}=3 / R \rho_{\mathrm{SiO} 2}$, with $R$ the mean particle radius (from SAXS) and $\rho_{\mathrm{SiO} 2}$ the density of silica $\left(2.2 \mathrm{~g} \mathrm{~cm}^{-3}\right)$.

\subsection{Surfactant adsorption onto Lys-Sil nanoparticles}

Adsorption isotherms of the surfactant $\mathrm{C}_{12} \mathrm{E}_{5}$ at the three Lys-Sil sols at $\mathrm{pH} 7$ and $20^{\circ} \mathrm{C}$ are shown in Fig. 2. The graphs present the surface concentration $\Gamma$ of the surfactant (amount adsorbed per unit area) $v s$. solution concentration expressed in the units of the critical micelle concentration $\left(\mathrm{cmc}=7 \times 10^{-5} \mathrm{M}\right.$ at $20^{\circ} \mathrm{C}$ ). The isotherms exhibit a steep increase in adsorption starting at an onset concentration below the $\mathrm{cmc}$, and a plateau value that is reached shortly above the $\mathrm{cmc}$. The isotherms can be represented by the S-type isotherm equation by $\mathrm{Gu}$ and $\mathrm{Zhu},{ }^{20}$

$$
\Gamma=\Gamma_{\mathrm{m}} \frac{K(c / \mathrm{cmc})^{n}}{1+(c / \mathrm{cmc})^{n}}
$$

where $\Gamma_{\mathrm{m}}$ represents the maximum surface concentration (plateau value of the adsorption isotherm), $K$ is the adsorption constant and $n$ is nominally the aggregation number of surface micelles. Fits of eqn (1) to the adsorption data are shown by the full curves in Fig. 2 and the parameters are summarized in Table 2. Also given is the surface aggregation concentration $c_{0}$, which can be calculated from the parameters $K$ and $n$ by eqn (2): ${ }^{20}$

Table 1 Parameters for synthesis and characterization of three Lys-Sil samples: stirring rate $r$ of the reaction mixture at temperature $T$, mean particle radius $R$ and size polydispersity $s$ as derived from SAXS, and specific surface area $a_{\mathrm{BET}}$ of the sols as determined from the nitrogen adsorption measurements ${ }^{a}$

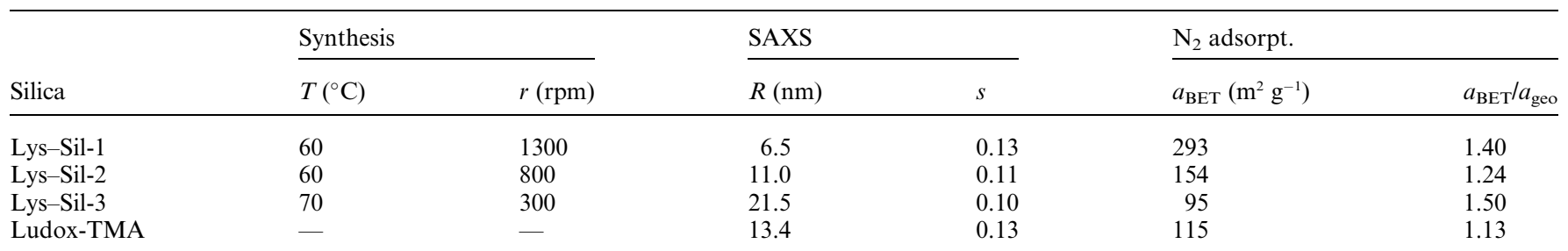

${ }^{a}$ Polydispersity is expressed by the standard deviation from the mean particle size; $a_{\text {geo }}$ is the geometric surface area. 


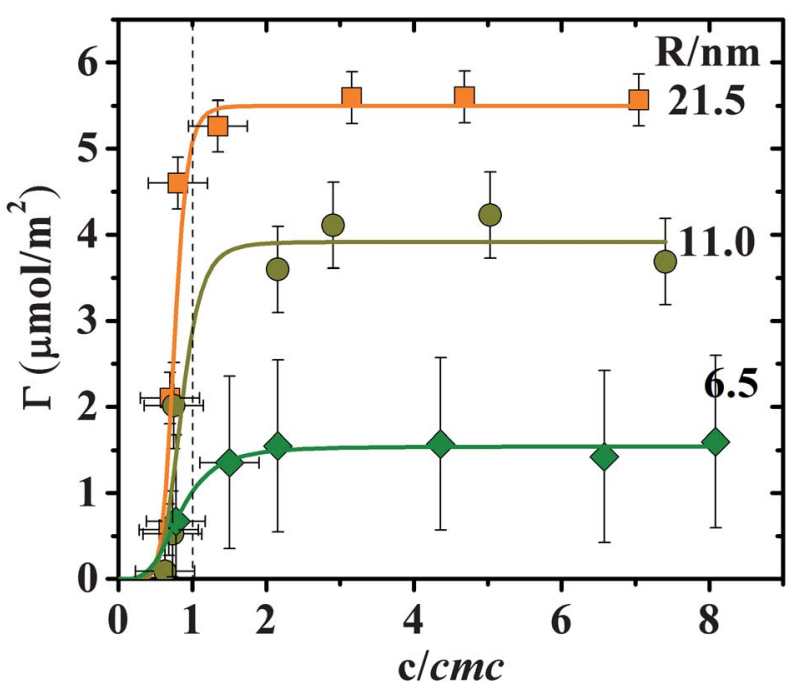

Fig. 2 Adsorption isotherms $\left(20^{\circ} \mathrm{C}\right)$ of the surfactant $\mathrm{C}_{12} \mathrm{E}_{5}$ at Lys-Sil silica particles of radius $6.5,11$ and $21.5 \mathrm{~nm}$ : experimental data and fits by the $\mathrm{Gu}-\mathrm{Zhu}$ equation. The dashed vertical line indicates the $\mathrm{cmc}$ of the surfactant.

Table 2 Adsorption of $\mathrm{C}_{12} \mathrm{E}_{5}$ at Lys-Sil nanoparticles. Fit of adsorption data of Fig. 2 by eqn (1): maximum surface concentration $\Gamma_{\mathrm{m}}$, adsorption constant $K$, nominal aggregation number of surface micelles $n$, and ratio of surface aggregation concentration to critical micelle concentration ${ }^{a}$

\begin{tabular}{llllll}
\hline Silica & $R(\mathrm{~nm})$ & $\Gamma_{\mathrm{m}}\left(\mu \mathrm{mol} \mathrm{m}{ }^{-2}\right)$ & $K$ & $n$ & $c_{0} / \mathrm{cmc}$ \\
\hline Lys-Sil-1 & 6.5 & 1.5 & 1.9 & 3.7 & 0.42 \\
Lys-Sil-2 & 11 & 3.9 & 2.8 & 6.2 & 0.58 \\
Lys-Sil-3 & 21.5 & 5.5 & 12 & 8.8 & 0.58 \\
${ }^{a}$ cmc of $\mathrm{C}_{12} \mathrm{E}_{5}$ in water is found to be $7 \times 10^{-5} \mathrm{M}$. & & \\
\hline
\end{tabular}

$$
c_{0} / \mathrm{cmc}=\left(\frac{n-1}{n+1}\right)^{(n+1) / n} K^{-1 / n}
$$

As indicated in Fig. 2, the uncertainty in the experimental values of $\Gamma_{\mathrm{m}}$ is large for the smallest particles. The uncertainty in $c_{0} / \mathrm{cmc}$ is estimated to $\pm 20 \%$.

The most interesting aspect of the adsorption isotherms in Fig. 2 is the strong decrease of the limiting surface concentration $\Gamma_{\mathrm{m}}$ with decreasing size of the Lys-Sil particles. In preliminary adsorption measurements for $\mathrm{C}_{12} \mathrm{E}_{5}$ on pure siliceous LudoxTMA particles $(R=13.4 \mathrm{~nm})$ we found a plateau value $\Gamma_{\mathrm{m}}=$ $4.5 \mathrm{mmol} \mathrm{m}{ }^{-2}$, which fits into the size dependence of $\Gamma_{\mathrm{m}}$ observed with the Lys-Sil particles.

Yokoi et al. ${ }^{21}$ investigated the formation and properties of Lys-Sil nanoparticles using a combination of liquid-state ${ }^{13} \mathrm{C}$ NMR, solid-state ${ }^{13} \mathrm{C} \mathrm{CP/MAS} \mathrm{NMR,} \mathrm{thermogravimetry,} \mathrm{and}$ differential thermal analysis. They concluded that a substantial fraction of lysine used in the particle synthesis remains adsorbed at the nanospheres. To find out in what way adsorbed lysine may affect the adsorption of the surfactant we studied the adsorption of lysine onto pure siliceous nanoparticles.

\subsection{Lysine adsorption onto silica nanoparticles}

The adsorption of lysine was studied on a Ludox-TMA silica sol, as this material is free from any other organic base. The results are shown in Fig. 3. Within error limits the adsorption data can be represented by the Langmuir equation, $\Gamma^{\mathrm{L}}=\Gamma_{\mathrm{m}}^{\mathrm{L}} b c /(1+b c)$, where $\Gamma^{\mathrm{L}}$ is the surface concentration of lysine at equilibrium concentration $c$. We find a limiting surface concentration $\Gamma_{\mathrm{m}}^{\mathrm{L}}=$ $1.8 \mu \mathrm{mol} \mathrm{m} \mathrm{m}^{-2}$ (corresponding to a surface density of $1.1 \mathrm{~nm}^{-2}$ ), and adsorption constant $b=1.6 \mathrm{mM}^{-1}$. Our value of the limiting surface density is consistent with the value of $0.5 \mathrm{~nm}^{-2}$ reported by Yokoi et al. ${ }^{21}$ for the specific conditions of their particle synthesis. From here on, the amount of lysine adsorbed at silica nanoparticles will be expressed by the relative surface concentration $\theta=\Gamma^{\mathrm{L}} / \Gamma_{\mathrm{m}}^{\mathrm{L}}$ to avoid mix-up with the adsorption of the surfactant.

\subsection{SANS study of surfactant aggregate structures}

SANS measurements were made to study surfactant aggregate structures at silica nanoparticles in the absence and presence of lysine. As in the preceding studies, ${ }^{14,15}$ SANS measurements were made with a $\mathrm{H}_{2} \mathrm{O}-\mathrm{D}_{2} \mathrm{O}$ mixture that matches the scattering length density of the silica. In this contrast-match scenario the silica particles become invisible to the neutron beam. Hence in the absence of surfactant only a constant scattering background is observed, as shown in the inset in Fig. 4a. When a surfactant is added, a scattering intensity profile $I(q)$ appears which is characteristic of the shape and size of the surfactant aggregates and their arrangement in space. Fig. $4 \mathrm{a}$ shows the scattering profile for $\mathrm{C}_{12} \mathrm{E}_{5}$ in a $3.3 \mathrm{wt} \%$ Ludox-TMA silica dispersion at a surfactant concentration corresponding to $\Gamma=3.5 \mu \mathrm{mol} \mathrm{m}{ }^{-2}$

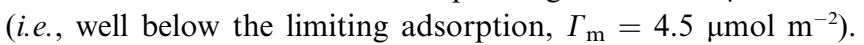
Also shown in Fig. 4a is the scattering profile of the surfactant in the absence of silica, measured at a similar concentration but in pure $\mathrm{D}_{2} \mathrm{O}$, to enhance the scattering contrast. The scattering curve for $\mathrm{C}_{12} \mathrm{E}_{5}$ in the absence of silica can be represented quantitatively by the form factor model of wormlike micelles. The resulting fit parameters are given in Table 3 .

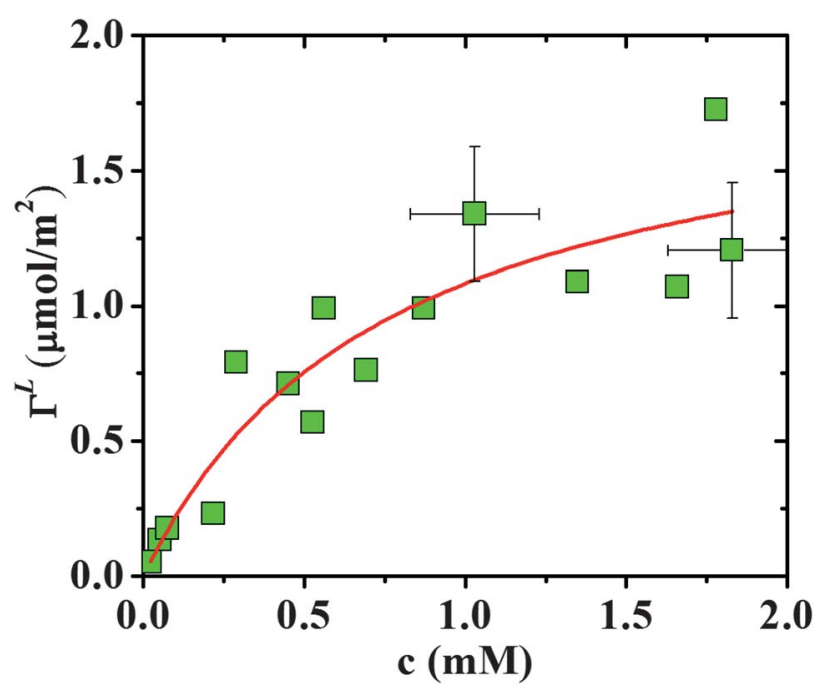

Fig. 3 Adsorption isotherm of lysine on Ludox-TMA silica: experimental data and fit by the Langmuir equation. 


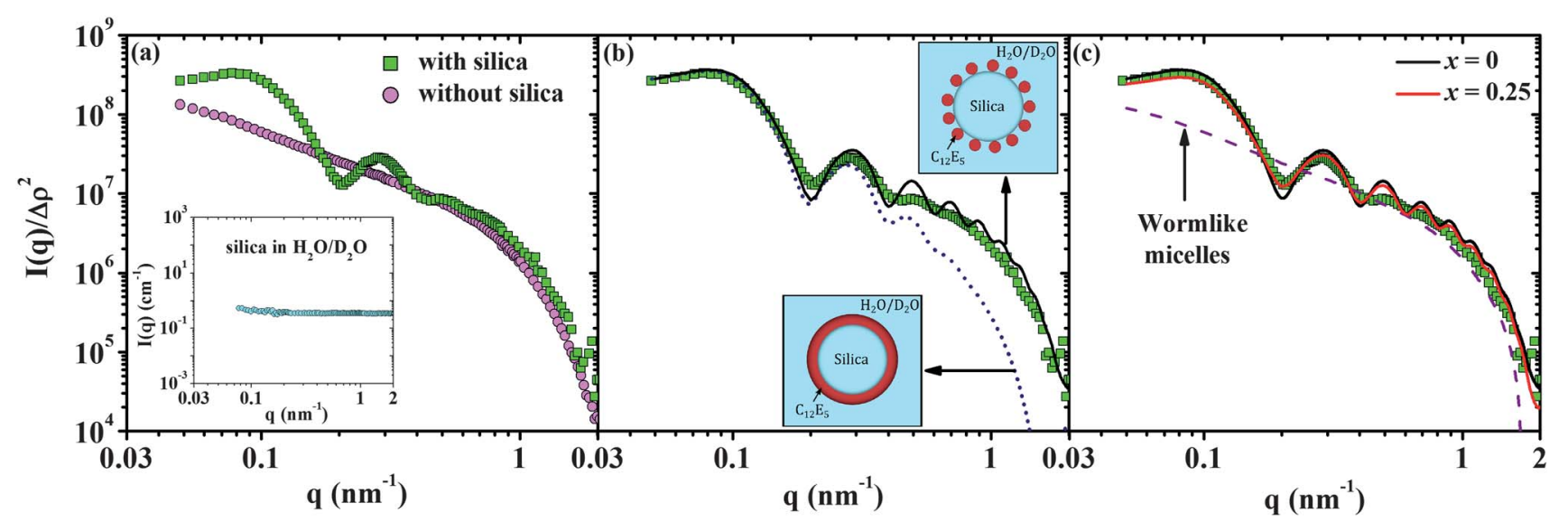

Fig. 4 SANS intensity profiles $I(q)$ for the surfactant $\mathrm{C}_{12} \mathrm{E}_{5}$ in a $3.3 \mathrm{wt} \%$ Ludox-TMA dispersion $\left(I=3.5 \mu \mathrm{mol} \mathrm{m}{ }^{-2}\right)$ at silica/water contrast match conditions: (a) comparison of scattering profiles in the presence of silica particles (squares) and the corresponding amount of surfactant in the absence: of silica particles (circles) (the inset shows the contrast match of silica in absence of surfactant); (b) fit of the scattering curve by the shell model (dotted curve) and the micelle-decorated bead model (full curve), with $R_{\mathrm{m}}=2.2$ and $N=105$; (c) fit to the scattering curve accounting for the dual population of the $25 \%$ surfactant as wormlike bulk micelles (red line) and rest adsorbed; the dashed curve in the graph is the form factor of the wormlike micelle.

Table 3 Analysis of SANS data for $\mathrm{C}_{12} \mathrm{E}_{5}$ adsorbed onto Ludox-TMA particles by the micelle-decorated bead model and spherical shell model ${ }^{a}$

\begin{tabular}{lllllll}
\hline & $\Gamma\left(\mu \mathrm{mol} \mathrm{m}{ }^{-2}\right)$ & $R_{\text {bead }}(\mathrm{nm})$ & $R_{\mathrm{m}}(\mathrm{nm})$ & $\Delta \rho\left(\times 10^{-4} \mathrm{~nm}^{-2}\right)$ & $N_{\text {mic }}$ & $L(\mathrm{~nm})$ \\
\hline Surf-micelles & 3.5 & 13.37 & 2.2 & 2.36 & 105 & - \\
Shell & 3.5 & 13.37 & - & 2.36 & - & \\
${ }^{a} R_{\mathrm{m}}$ is the radius of surface micelles and $L$ is the thickness of shell according to core shell model. & & \\
\hline
\end{tabular}

The scattering profile of the surfactant in contact with silica particles was analysed in terms of two different models: (i) a spherical shell model, ${ }^{12}$ assuming that the surfactant is forming a layer of uniform thickness $L$, and (ii) the micelle-decorated bead model, ${ }^{22}$ assuming a random distribution of $N$ spherical surface micelles of radius $R_{\mathrm{m}}$ located at a distance $R+R_{\mathrm{m}}$ from the center of the silica particle. The parameters used to fit the data are given in Table 3. Fig. $4 \mathrm{~b}$ shows that both models give a fair representation of the experimental data in the low- $q$ regime including the local maximum near $q=0.3 \mathrm{~nm}^{-1}$. For higher $q$ the shell model predicts a steeper decrease of $I(q)$ than the observed scattering curve. This deviation indicates that the shell model underestimates the overall surface area of the surfactant aggregates. The micelle-decorated bead model gives a satisfactory fit of the entire scattering curve. The higher-order oscillations in the region of $q>0.4 \mathrm{~nm}^{-1}$ produced by this model are caused by artifacts arising from the Fourier transformation of the paircorrelation function of surface micelles to derive the intermicellar structure factor. This pair-correlation function was generated by simulating random distributions of $N$ spherical micelles on the silica bead (see ESI $\dagger$ ). We stress that we have not attempted to determine the detailed shape of the surface micelles, but the model of spherical surface micelles was adopted for the sake of simplicity. However, we have tested if the fit can be further improved by assuming that a part of the surfactant is not adsorbed but exists in the form of free wormlike micelles. Specifically, the total scattering intensity was represented by

$$
I(q)=(1-x) I_{\text {surf }}(q)+x I_{\text {bulk }}(q)
$$

where $I_{\text {surf }}(q)$ and $I_{\text {bulk }}(q)$ represent the scattering intensity functions of the micelle-decorated bead model and the model of free cylindrical micelles, respectively, and $x$ is the fraction of scattering intensity contributed by the free micelles. Fig. 4c shows that eqn (3) with $x=0.25$ gives indeed some improvement of the fit. However, more systematic studies and consideration of the finite experimental resolution of the SANS data (experimental smearing) would be necessary to discriminate between the small differences of the two models. The limitations of eqn (3) to account for the co-existence of two populations of surfactant micelles in the system are discussed below.

To establish the effect of lysine on the aggregate structure of the surfactant in the silica dispersion, SANS measurements were made for a set of samples of fixed concentration of $\mathrm{C}_{12} \mathrm{E}_{5}$ $(20 \mathrm{mM})$ in $3.3 \mathrm{wt}^{\%} \%$ dispersions of Ludox-TMA and with gradually increasing concentrations of lysine. From the known adsorption isotherm of lysine on Ludox-TMA (Fig. 3) its amount in the samples was adjusted such as to cover a wide range of surface concentrations while keeping the concentration of free lysine in the solution as low as possible $(c<3 \mathrm{mM})$. Scattering profiles for lysine surface concentrations $\theta$ from 0.004 to 0.76 are presented in Fig. 5a. It can be seen that the peak in $I(q)$ at $q \approx$ $0.3 \mathrm{~nm}^{-1}$, which is a measure of the amount of surfactant forming the adsorbed layer, decreases in height as the lysine concentration at the surface increases. A small peak is still detectable at a lysine surface concentration $\theta=0.62$, but the peak has vanished

\# The scattering curve for $\mathrm{C}_{12} \mathrm{E}_{5}$ without silica was measured in pure $\mathrm{D}_{2} \mathrm{O}$ to enhance the contrast. 


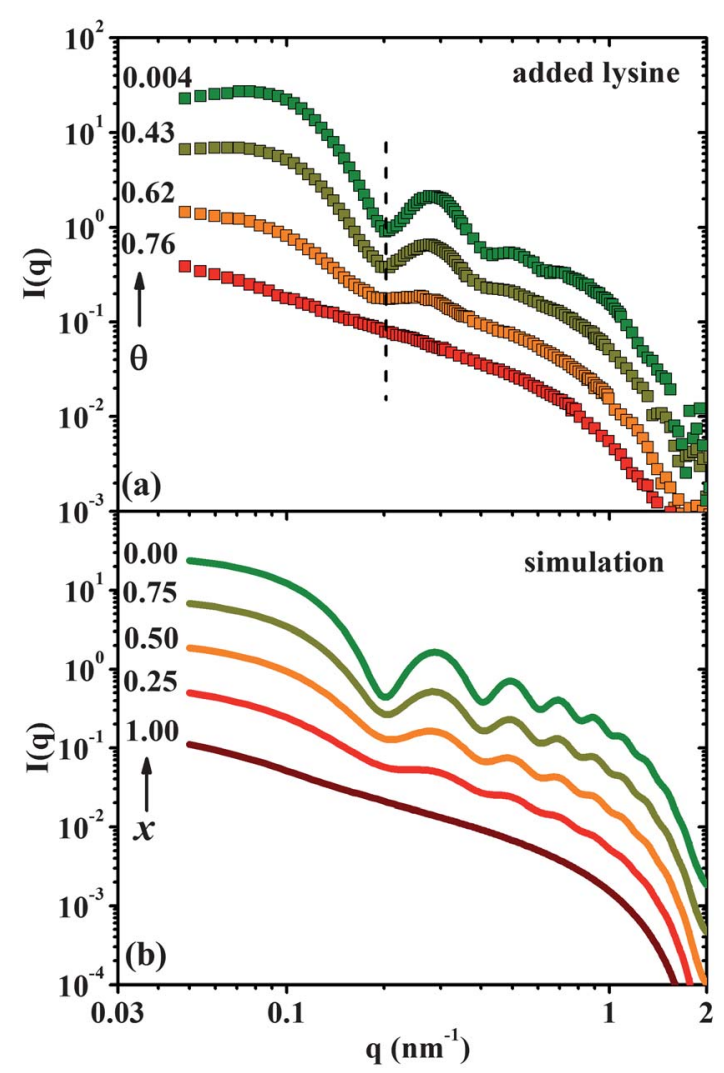

Fig. 5 (a) SANS experimental scattering curves for $\mathrm{C}_{12} \mathrm{E}_{5}$ in a $3.3 \mathrm{wt} \%$ dispersion of Ludox-TMA with increasing lysine concentration corresponding to relative surface concentrations $\theta$ from 0.004 to 0.76 ; (b) simulation of data in (a) based on eqn (3) with weight factor $x$ of bulk micelles as given in the graph. In (a) and (b) curves for higher $\theta$ or $x$ are shifted downward by factors of 3 .

at $\theta=0.76$. The scattering profile at this highest lysine concentration resembles the profile obtained for the surfactant in the absence of silica ( $c f$. Fig. 4a). This clearly indicates that at $\theta>0.75$ all surfactant has been displaced from the surface of the silica particles and is forming wormlike micelles in the aqueous phase. This conclusion is justified as it was confirmed by SANS measurements that lysine does not affect the scattering profile of $\mathrm{C}_{12} \mathrm{E}_{5}$ in the absence of the silica (not shown), and hence has no significant influence on the morphology of the bulk micellar aggregates at the lysine concentrations relevant in the present context. Quantitative modelling of the scattering profiles in terms of two co-existing populations of surfactant aggregates (surface micelles and bulk aggregates) in the presence of lysine was not practicable because too many of the relevant parameters were unknown. Instead, we checked if the gradual change of the scattering profile as a function of added lysine can be represented by eqn (3), i.e., an incoherent superposition of contributions from surface micelles and free cylindrical micelles as in the bulk solution. Results for $x=0$ (all surfactant forming spherical surface micelles), $x=1$ (all surfactant forming wormlike micelles in solution) and intermediate states with $x=0.25,0.5$ and 0.75 are shown in Fig. 5b. The parameters used for simulating individual form factors of a silica bead with adsorbed surface micelles $\left(P_{\text {surf-mic }}(q)\right)$ and free wormlike micelles in the bulk $\left(P_{\text {worm }}(q)\right)$ are given in Table 4 . It can be seen that the simulated curves reproduce the trends of the experimental scattering profiles as a function of $\theta$ (Fig. 5a).

Closer inspection of the SANS profiles in Fig. 5a reveals that the main oscillation in $I(q)$ shifts to lower values of $q$ as the surface concentration $\theta$ of lysine increases. In terms of the simple core-shell model, this shift corresponds to an increase in the mean layer thickness from $4 \mathrm{~nm}$ to roughly $6 \mathrm{~nm}$. This suggests that small surface concentrations of lysine are causing only partial detachment of the oxyethylene head groups of the surfactant, connected with a rehydration of these groups. Hence the lysine-induced weakening of the binding of surfactant to the surface may involve the formation of a water-rich layer between the surfactant aggregates and the surface, which ultimately leads to the complete detachment and reorganisation of the surfactant aggregates. This finding is reminiscent of the effect of temperature on the layer of $\mathrm{C}_{12} \mathrm{E}_{5}$ at silica particles as reported by Cummins et al., ${ }^{12}$ which was attributed to a temperature-induced reorganization and partial desorption of the surfactant.

\section{Discussion}

\subsection{Size dependence of the adsorption of $\mathrm{C}_{12} \mathrm{E}_{5}$ on Lys-Sil}

The sigmoidal shape of the adsorption isotherms in Fig. 2 is a well-known signature of aggregative adsorption of nonionic surfactants at hydrophilic surfaces..$^{6,9,11,20}$ On the other hand, the strong decrease of the maximum surface concentration of the surfactant with decreasing size of the Lys-Sil nanoparticles represents a remarkable new result. To our knowledge such a pronounced size effect on the adsorption has not been reported previously. This may be due to the difficulty of preparing oxide nanoparticles of well-defined size in this size range and determining the precise concentration of surfactant in the dispersion. Here we will discuss this finding from a point of view of the different structures of surfactant aggregates at the surface of the nanoparticles and in solution.

The SANS measurements presented in Section 2.4 have established that $\mathrm{C}_{12} \mathrm{E}_{5}$ is forming discrete surface aggregates at the Ludox-TMA silica particles, in agreement with our earlier findings with $\mathrm{C}_{12} \mathrm{E}_{5}$ at Stöber-type silica nanoparticles of similar size $(16 \mathrm{~nm}) .{ }^{14}$ In that paper we conjectured that surface micelles are preferred because the high surface curvature of small particles prevents an effective packing of surfactant molecules in a bilayer film. For a particle of radius $R$ with an adsorbed bilayer film of thickness $L$ the area at the midpoint plane of the bilayer exceeds the surface area of the particle by a factor $f=(1+$ $L / 2 R)^{2}$. For the present Lys-Sil particles and a bilayer thickness of $4 \mathrm{~nm}$ we have $f=1.2$ for the largest particles $(R=21.5 \mathrm{~nm})$, but $f=1.7$ for the smallest particles $(R=6.5 \mathrm{~nm})$. For large particles this curvature-induced handicap may be met by formation of an asymmetric bilayer, having a higher number of molecules in the outer layer. For the smallest particles, on the other hand, it appears that the curvature-induced handicap is too high for any form of bilayer structure. Instead, self-assembly apparently leads to discrete, more highly curved surface aggregates, as indicated by the SANS study. As shown above for $\mathrm{C}_{12} \mathrm{E}_{5}$ at Ludox-TMA particles, the data can be represented by spherical surface micelles of about $4.4 \mathrm{~nm}$ in diameter. Assuming that this also pertains to $\mathrm{C}_{12} \mathrm{E}_{5}$ adsorbed at the Lys-Sil particle, we 
basic terminal amino group of lysine. Accordingly, adsorption of lysine causes a decrease in the number density of free silanol groups at the silica particles and thus a weaker binding of the surface micelles. As can be seen in Fig. 5, the increase in the surface concentration $\theta$ of lysine (Fig. 5a) indeed correlates directly with the fraction $x$ of the displaced surfactant (Fig. 5b).

Finally, we discuss the possible influence of lysine on the size dependence of the adsorption of $\mathrm{C}_{12} \mathrm{E}_{5}$ at the Lys-Sil nanoparticles (Fig. 2). Since the three samples of Lys-Sil were synthesized from the same reaction mixture, and particle size was tuned solely by the stirring rate and a weak temperature increase (see Table 1), we believe that the surface density of lysine and silanol groups was independent of particle size. This is in line with earlier published results on similar silica particles ${ }^{23}$ where the surface density of lysine on the Lys-Sil particles was estimated to be $0.5 \mathrm{~nm}^{-2}$, and a value $3 \mathrm{~nm}^{-2}$ was adopted for the surface density of silanol groups as reported previously by Shenderovich et al. ${ }^{26}$ From these values the fraction of silanol groups blocked by lysine was estimated to be $15 \%$. This then implies that the surface density of free silanol groups at the LysSil particles will be lower than for pure Stöber-type or Ludoxtype silica. On the assumption that the binding strength of surface micelles of $\mathrm{C}_{12} \mathrm{E}_{5}$ is proportional to the surface density of free silanol, we may expect that the number of surface micelles on the Lys-Sil particles will be smaller than on Stöber-type or Ludox-type particles of equal size. Furthermore, we propose that this effect should be more pronounced for small, highly curved silica particles because in this case the straining energy would be higher than on larger particles. This conjecture is supported by the results of the earlier study of $\mathrm{C}_{12} \mathrm{E}_{5}$ at Stöber-type silica nanoparticles of radius $8 \mathrm{~nm},{ }^{14}$ for which a significantly higher limiting surface concentration was found than on the present Lys-Sil particles of radius $11 \mathrm{~nm}$. However, more systematic work is needed to corroborate this combined influence of particle size and density of binding sites.

\subsection{Potential application relevance}

The finding that a nonionic surfactant can be displaced readily from the surface of nanoparticles by small amounts of a more strongly adsorbed substance is of practical relevance for the formulation of dispersions stabilized by such surfactants. In addition, the displaced surfactant can cause significant changes in the macroscopic behaviour of the system. Since non-ionic surfactants like $\mathrm{C}_{12} \mathrm{E}_{5}$ are forming wormlike micelles in a wide temperature and concentration range their displacement from the surface can affect the rheological behaviour of the system. ${ }^{27}$ For these surfactants it is even possible that the displacement of the surfactant drives the system from the one-phase region to the two-phase region of the surfactant + water phase diagram. For the present system this is demonstrated in Fig. 6. The phase diagram of the $\mathrm{C}_{12} \mathrm{E}_{5}+$ water exhibits a lower critical point (cloud point) at about $31{ }^{\circ} \mathrm{C}$ and $1 \mathrm{wt} \%$ surfactant. ${ }^{28}$ Above this temperature the system separates into a water-rich and a surfactant-rich phase. Fig. 6 a shows the turbidity $v$. temperature of a 1 $\mathrm{wt} \%$ Ludox-TMA dispersion containing $0.2 \mathrm{wt} \% \mathrm{C}_{12} \mathrm{E}_{5}$, without and with added lysine. In the absence of lysine almost all surfactant is adsorbed at the particles (surface concentration $\Gamma=$ $4 \mu \mathrm{mol} \mathrm{m}{ }^{-2}$, i.e., close to the limiting adsorption). The turbidity

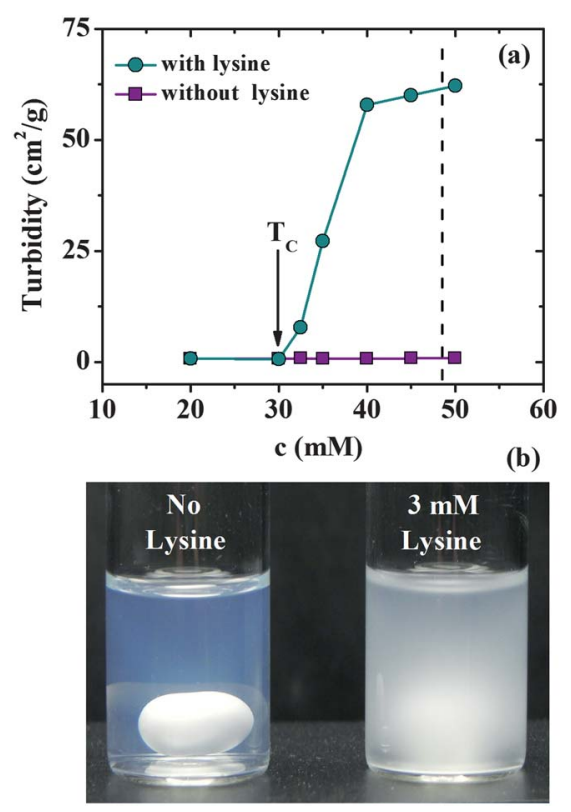

Fig. 6 (a) Turbidity of $5.2 \mathrm{mM} \mathrm{C}_{12} \mathrm{E}_{5}$ in a $1 \mathrm{wt} \%$ dispersion of LudoxTMA without lysine (squares) and with $3 \mathrm{mM}$ lysine (circles); (b) photograph of the two constantly stirred samples at a temperature well above the cloud point $T_{\mathrm{c}}$ (dashed line in (a)).

of this sample is moderately low and independent of temperature up to $50{ }^{\circ} \mathrm{C}$. In the presence of lysine $(3 \mathrm{mM})$, however, when the surfactant is detached from the particles, the turbidity of the stirred sample strongly increases at temperatures $T>T_{\mathrm{c}}$, as most of the free surfactant is now forming droplets of the surfactantrich phase. The silica particles remain dispersed in the aqueous phase. SAXS measurements on the samples without and with lysine show no increased intensity in the Guinier regime at different temperatures, confirming the absence of any aggregation of silica nanoparticles. ${ }^{29}$ The scattering curves taken at temperatures from 20 to $50{ }^{\circ} \mathrm{C}$ superimpose (see ESI $\dagger$ ). Photographs of the samples without and with lysine at a temperature above $T_{\mathrm{c}}$ are shown in Fig. 6b. This is a striking example to show the potential significance of such displacement effects for the formulation of nanoparticle dispersions with nonionic surfactants. Related phenomena were reported recently by Mustafina et al. ${ }^{30}$

\section{Experimental}

\subsection{Materials}

$\mathrm{C}_{12} \mathrm{E}_{5}$ (Sigma-Aldrich, $\geq 98 \%$ ), 2,6-diaminohexanoic acid (lysine) (Fluka, purity $>98 \%$ ), tetraethyl orthosilicate (TEOS) (ABCR $\mathrm{GmbH}$, purity $>98 \%$ ) and $\mathrm{D}_{2} \mathrm{O}$ (Euriso-top, 99.9\%) were used without further purification. Water was purified by a MilliPore QPAK ${ }^{\circledR}(2)$ unit. Ludox-TMA (30 wt $\%$ suspension in water) was supplied by Sigma-Aldrich and further purified by dialysis and filtration through $0.22 \mu \mathrm{m}$ filters.

\subsection{Synthesis of silica nanoparticles}

Lys-Sil silica sols of different particle sizes were prepared by the hydrolysis of TEOS with water in the presence of the basic amino 
acid lysine, as reported by Davis et al. ${ }^{19}$ and Thomassen et al. ${ }^{31}$ Particle size was tuned by using pre-optimized stirring rates and temperature conditions of the reaction mixture (Table 1). The resulting silica dispersions were dialyzed to remove unreacted TEOS, lysine and reaction byproduct ethanol. Details of the synthesis and product clean-up are given elsewhere. ${ }^{29}$ The silica concentration of the resulting dispersions was determined gravimetrically ( $c a .3 \mathrm{wt} \%)$.

The silica sols were characterized by small-angle X-ray scattering (SAXS), nitrogen adsorption, and transmission electron microscopy (TEM). SAXS measurements were performed on a SAXSess $\mathrm{mc}^{2}$ instrument (Anton Paar, Austria) and the Saxsquant 3.50 software was used for data reduction and desmearing. Nitrogen adsorption isotherms at $77 \mathrm{~K}$ were determined on a Gemini III 2375 volumetric surface analyser (Micromeritics). For this purpose the dialysed silica dispersions were dried at 350 $\mathrm{K}$ for two days, then outgassed at $393 \mathrm{~K}$ for $1 \mathrm{~h}$ under vacuum, and finally reweighed to determine the precise mass of the sample. TEM images were taken by a FEI Technai $\mathrm{G}^{2} 20$ S-Twin electron microscope operating at $200 \mathrm{kV}$.

\subsection{Adsorption measurements}

Adsorption isotherms of $\mathrm{C}_{12} \mathrm{E}_{5}$ on the silica sols were determined with dilute dispersions $(0.1 \mathrm{wt} \%)$ by equilibration with appropriate amounts of the surfactant $(24 \mathrm{~h})$. After removal of the silica by centrifugation ( $4 \mathrm{~h}$ at $9500 \mathrm{~g}$ ) the concentration of nonadsorbed surfactant in the supernatant was determined by surface tension measurements. Surfactant concentrations above the $\mathrm{cmc}$ were determined by dilution with known amounts of water until the surface tension $\sigma$ attained a value somewhat higher than $\sigma_{\mathrm{cmc}}$. Systematic errors in the determination of the adsorbed amount in the plateau region of the adsorption isotherm can arise if not all the silica was removed by the centrifugation. In this case the surfactant can be desorbed from the particles in the dilution step, thus purporting a lower adsorption. Such an error will be largest for the smallest silica particles due to their slowest sedimentation in the centrifugation step. Systematic errors in the surface concentration $\Gamma$ of adsorbed surfactant can also arise from errors in the silica concentration of the dispersion (up to $\pm 5 \%$ ) and the specific surface area of the silica $( \pm 2 \%)$.

The extent of lysine binding at the silica surface was determined by adsorption measurements on Ludox-TMA. Lysine solutions of appropriate concentrations in water were prepared and a fixed volume of the silica dispersion was added. After equilibration $(24 \mathrm{~h})$ the silica sol was separated from the supernatant by centrifugation $(2 \mathrm{~h}$ at $21000 \mathrm{~g})$ and the residual concentration of lysine was determined by reaction with ninhydrin to Ruhman's purple which was detected by its absorbance at $570 \mathrm{~nm}$.

\subsection{SANS measurements}

Dispersions of Ludox-TMA (3.3 wt $\%$ ) were prepared in a $\mathrm{H}_{2} \mathrm{O}-$ $\mathrm{D}_{2} \mathrm{O}$ mixture with $61.7 \mathrm{wt} \% \mathrm{D}_{2} \mathrm{O}$, which has the same scattering length density (SLD) as silica (SLD $=3.54 \times 10^{-4} \mathrm{~nm}^{-2}$ ). A constant amount of $\mathrm{C}_{12} \mathrm{E}_{5}$ but increasing quantities of lysine were added to these silica dispersions. SANS measurements were carried out using the SANS-II instrument at the Paul Scherrer Institute, Villigen $(\mathrm{CH})$. Three different sample-to-detector distances were used to cover values of the scattering vector $q$ from 0.05 to $2.8 \mathrm{~nm}^{-1}$. The $2 \mathrm{D}$ scattering data were reduced to 1D profiles using the BerSANS software package. SciLab was used for building up codes of different models for further analysis of the scattering curves.

\section{Conclusions}

This study has shown that the self-assembly of surfactants in a dispersion of nanoparticles can be tuned by an additive which modifies the surface energy by adsorption onto the particles. Lysine acts as an effective surface modifier in the present system. Small concentrations of lysine cause a complete displacement of the surfactant $\mathrm{C}_{12} \mathrm{E}_{5}$ from silica particles. SANS measurements reveal that the displacement process represents a morphological transition from discrete surface micelles attached to the particles to elongated (wormlike) micelles in the aqueous bulk phase. The detachment seems to proceed via an intermediate state in which a hydrated layer of adsorbed lysine intercalates between the surfactant aggregates and the surface. Further SANS studies are needed to elucidate this process.

For Lys-Sil particles we find a pronounced decrease of the maximum surface concentration of the surfactant with decreasing particle size. Our study suggests that this size effect is caused by the adsorbed layer of lysine, which reduces the binding strength of the surfactant head groups, in combination with the increasing curvature of the solid surface. For the smallest particles (diameter $13 \mathrm{~nm}$ ) only a fraction of their surface is decorated with surface micelles. We speculate that this may be a consequence of a stronger distortion of surface micelles that is needed for attaining a sufficiently large contact area with the surface of small particles when some of the sites are blocked by lysine. The much higher limiting adsorption of the surfactant at the largest particles $(43 \mathrm{~nm})$ indicates that in this case the surfactant is not forming discrete surface micelles but less highly curved aggregates which allow a higher packing density at the surface. We present a simple model to account for such a transition in surface aggregate structure. Hence, this study can contribute to a better understanding of the factors controlling the self-assembly of surfactants at nanoparticles. This will be useful in the formulation of nanoparticle dispersions and their application in particle nanotechnology.

\section{Acknowledgements}

We wish to thank Dr D. M. Lugo and Dr S. Prevost for their support in the data analysis and interpretation, and S. Selve (ZELMI, TU Berlin) for help with TEM measurements. This work is based on experiments performed at the Swiss spallation neutron source SINQ, Paul Scherrer Institute, Villigen, Switzerland, and has been supported by the European Commission under the $7^{\text {th }}$ Framework Programme through the 'Research Infrastructures' action of the 'Capacities' Programme, contract no: CP-CSA_INFRA-2008-1.1.1 number 226507-NMI3. Financial support by the German Research Foundation (DFG) in the framework of IGRTG 1524 is also gratefully acknowledged. 


\section{Notes and references}

1 Handbook of Applied Surface and Colloid Chemistry, ed. K. Holmberg, D. O. Shah and M. J. Schwuger, John Wiley, New York, 2002; S. Paria and K. C. Khilar, J. Colloid Interface Sci., 2004, 110, 75; L. K. Limbach, R. Bereiter, E. Müller, R. Krebs, R. Gälli and W. J. Stark, Environ. Sci. Technol., 2008, 42, 5828; N. Veronovoski, P. Andeozzi, C. L. Mesa, M. S. Smol and V. Ribitsch, Colloid Polym. Sci., 2010, 288, 387; R. Zhang and P. Somasundaran, Adv. Colloid Interface Sci., 2006, 123-126, 213.

2 N. A. Klimenko and A. M. Koganovskii, Kolloidn. Zh., 1974, 36, 151 N. A. Klimenko, V. L. Kofanov and E. G. Sivalov, Kolloidn. Zh., 1981, 43, 287.

3 P. Levitz, Langmuir, 1991, 7, 1595.

4 M. R. Böhmer, L. K. Koopal, R. Janssen, E. M. Lee, R. K. Thomas and A. R. Rennie, Langmuir, 1992, 8, 2228.

5 S. Manne and H. E. Gaub, Science, 1995, 270, 1480.

6 F. Tiberg, J. Chem. Soc., Faraday Trans., 1996, 92, 531.

7 L. M. Grant, F. Tiberg and W. A. Ducker, J. Phys. Chem. B, 1998, 102, 4288.

8 R. Steitz, P. Müller-Buschbaum, S. Schemmel, R. Cubitt and G. H. Findenegg, Europhys. Lett., 2004, 67, 962.

9 O. Dietsch, A. Eltekov, H. Bock, K. E. Gubbins and G. H. Findenegg, J. Phys. Chem. C, 2007, 111, 16045.

10 J. Penfold, E. Staples and I. Tucker, Langmuir, 2002, 18, 2967.

11 M. K. Matsson, B. Kronberg and P. M. Claesson, Langmuir, 2004, 20, 4051.

12 P. G. Cummins, J. Penfold and E. Staples, J. Phys. Chem., 1992, 96, 8092.

13 J. Penfold, E. Staples, I. Tucker and P. Cummins, J. Phys. Chem., 1996, 100, 18133.

14 D. M. Lugo, J. Oberdisse, M. Karg, R. Schweins and G. H. Findenegg, Soft Matter, 2009, 5, 2928.

15 D. M. Lugo, J. Oberdisse, A. Lapp and G. H. Findenegg, J. Phys. Chem. B, 2010, 114, 4183.
16 K. P. Sharma, V. K. Aswal and G. Kumaraswami, J. Phys. Chem. B, 2010, 114, 10986.

17 S. Kumar and V. K. Aswal, J. Phys.: Condens. Matter, 2011, 23, 035101.

18 S. Ahualli, G. R. Iglesias, W. Wachter, M. Dulle, D. Minami and O. Glatter, Langmuir, 2011, 27, 9182.

19 T. M. Davis, M. A. Snyder, J. E. Krohn and M. Tsapatsis, Chem. Mater., 2006, 18, 5814.

20 T. Gu and B.-Y. Zhu, Colloids Surf., 1990, 44, 81; B.-Y. Zhu, T. Gu and X. Zhao, J. Chem. Soc., Faraday Trans. 1, 1989, 85, 3819.

21 T. Yokoi, J. Wakabayashi, Y. Otsuka, W. Fan, M. Iwama, R. Watanabe, K. Aramaki, A. Shimojima, T. Tatsumi and T. Okubo, Chem. Mater., 2009, 21, 3719.

22 G. Despert and J. Oberdisse, Langmuir, 2003, 19, 7604; J. Oberdisse, Phys. Chem. Chem. Phys., 2004, 6, 1557.

23 R. Lipowsky and H.-G. Döbereiner, Europhys. Lett., 1998, 43, 219.

24 S. Partyka, S. Zaini, M. Lindeheimer and B. Brun, Colloids Surf., 1984, 12, 255; P. Trens and R. Denoyel, Langmuir, 1993, 9, 519.

25 D. Müter, T. Shin, B. Demé, P. Fratzl, O. Paris and G. H. Findenegg, J. Phys. Chem. Lett., 2010, 1, 1442.

26 I. G. Shenderovich, D. Mauder, D. Akcakayiran, G. Buntkowsky, H.-H. Limbach and G. H. Findenegg, J. Phys. Chem. B, 2007, 111, 12088.

27 R. Strey, Ber. Bunsenges. Phys. Chem., 1996, 100, 182.

28 R. Strey, R. Schomäcker, D. Roux, F. Nallet and U. Olsson, J. Chem. Soc., Faraday Trans., 1990, 86, 2253.

29 B. Bharti, J. Meissner and G. H. Findenegg, Langmuir, 2011, 27, 9823.

30 A. R. Mustafina, J. G. Elistratova, O. D. Bochkova, V. A. Burilov, S. V. Fedorenko, A. I. Konovalov and S. Y. Soloveva, J. Colloid Interface Sci., 2011, 354, 644.

31 L. C. J. Thomassen, A. Aerts, V. Rabolli, D. Lison, L. Gonzalez, M. Kirsch-Volders, D. Napierska, P. H. Hoet, C. E. A. Kirschhock and J. A. Martens, Langmuir, 2010, 26, 328. 
Addition and correction

View Article Online

\section{Note from RSC Publishing}

This article was originally published with incorrect page numbers. This is the corrected, final version.

The Royal Society of Chemistry apologises for these errors and any consequent inconvenience to authors and readers. 